\title{
Beyond strain release: Delocalisation-enabled organic reactivity
}

\author{
Alistair J. Sterling, ${ }^{1}$ Russell C. Smith, ${ }^{2}$ Edward A. Anderson ${ }^{3, *}$ \& Fernanda Duarte ${ }^{3, *}$
}

\begin{abstract}
Strain energy has long been recognised as a fundamental driving force for organic reactions. However, the release of strain alone is an insufficient predictor of reactivity, as seen in the equivalent strain energies but disparate reactivity of cyclopropane and cyclobutane. Here we show that electronic delocalisation is a key factor that operates alongside strain release to boost reactivity, significantly lowering the energy required for bond-breaking in cyclopropanes, cycloalkynes and cycloalkenes. Consideration of thermodynamic and delocalisation parameters explains the relative rates of reaction of molecules containing these functional groups, leading to a 'hierarchy of delocalisation' and a rule-of-thumb model that accurately predicts activation barriers. The implications of these principles are demonstrated in the context of the reactions of strained building blocks commonly encountered in total synthesis, medicinal chemistry, polymer science and bioconjugation.
\end{abstract}

Main text. The release of strain energy has long been harnessed as a fundamental driving force in organic synthesis. For instance, 'ring strain' in three- and four-membered rings, one of the basic tenets of undergraduate chemistry, ${ }^{1}$ imparts heightened reactivity through deviations from ideal bond angles - so-called Baeyer strain. ${ }^{2}$ Accordingly, 'strain release' is often deployed as a powerful tactic in organic synthesis as a means to increase reaction rates (Fig. $1 \mathrm{a})$; this strategy has found applications in total synthesis, ${ }^{3}$ polymer science,${ }^{4,5}$ bioconjugation, ${ }^{6,7}$ and the synthesis of bioisosteres in drug design, ${ }^{8}$ and is also an important concept in biosynthesis. $^{9}$

Despite the prevailing dogma that pent-up strain energy explains the reactivity of small rings, even the simplest of these systems presents a paradox: cyclopropanes display markedly heightened ring-opening reactivity over cyclobutanes $\left(k_{\text {rel }}=10^{4}-10^{7}\right.$ for intramolecular ring opening reactions), ${ }^{10}$ despite the two molecules possessing nearly identical strain energies (27.5 and $26.5 \mathrm{kcal} \mathrm{mol}^{-1}$, respectively). ${ }^{2}$ Even more marked is a comparison with ring-opening reactions of the inter-bridgehead bond in [1.1.1]propellane (1), which is many orders of magnitude more reactive than cyclopropane, again despite the release of a similar amount of strain energy ( $30 \mathrm{kcal} \mathrm{mol}^{-1}$, Fig. 1a) ${ }^{11}$

This puzzle has been the subject of decades of theoretical investigation. Stirling et al. ${ }^{12}$ proposed a classical steric explanation, concluding that a larger proportion of angle strain is relieved in cyclopropane $(\sim 75 \%)$ than the equivalent process for cyclobutane $(\sim 50 \%)$. The groups of $\mathrm{Hoz}^{13}$ and Houk ${ }^{14}$ argued that differences in electronic structure (i.e., bonding) are

\footnotetext{
${ }_{1}^{1}$ Physical and Theoretical Chemistry Laboratory, South Parks Road, Oxford, OX1 3QZ, UK

${ }^{2}$ Abbvie Drug Discovery Science \& Technology (DDST), 1 North Waukegan Road, North Chicago, IL 60064, USA

${ }^{3}$ Chemistry Research Laboratory, Mansfield Road, Oxford, OX1 3TA, UK.

E-mail: edward.anderson@chem.ox.ac.uk, fernanda.duartegonzalez@chem.ox.ac.uk
} 
instead the cause of the reactivity difference: Hoz proposed that deformation-induced rehybridisation enhances the electrophilicity of $\mathrm{C}-\mathrm{C}$ bonds by lowering the energy of the lowest unoccupied molecular orbital (LUMO) of the breaking bond, while Houk invoked an orbital interactions through-bonds (OITB) $)^{15}$ argument in which transition state (TS) aromaticity stabilises ring-opening reactions of cyclopropane, in comparison with antiaromatic TS destabilisation for equivalent reactions of cyclobutane.

That the electronic structure of cyclopropane is linked to its distinct reactivity profile can be connected to the commonly used bonding models for cyclopropane. For example, the Coulson-Moffitt 'bent bonds' description, ${ }^{16}$ Dewar's $\sigma$-aromaticity proposal, ${ }^{17}$ and Weinhold and Landis' geminal hyperconjugation model ${ }^{18}$ all indicate greater electronic delocalisation of the $\mathrm{C}-\mathrm{C}$ bonds in the ground state, whereby the bonding electron pair is partially delocalised around the three-membered ring (Fig. $1 \mathrm{~b}$ ). In contrast, the 'ordinary' $\mathrm{C}-\mathrm{C} \sigma$-bonds of cyclobutane are essentially localised. In this work, we employ this delocalisation concept to propose a link between bonding, strain energy and reactivity (Fig. 1c). We propose that an earlier, lower energy TS arises from enhanced delocalisation of the electrons from breaking bonds within three-membered rings, complementing the barrier-lowering effect arising from strain release. This general model explains not only the relative reactivity of cyclopropane compared with cyclobutane, but that of any molecule containing one or more three-membered rings, including heterocycles and polycyclic structures. For example, we show that the well-defined reactivity of highly-strained bicyclo[1.1.0]butanes and [1.1.1]propellane follows naturally from their ability to undergo electronic delocalisation. ${ }^{19}$ Similarly, we propose that C-O delocalisation in epoxides explains their far greater ring-opening reactivity than oxetanes, which are employed as chemically inert bioisosteres for carbonyl groups. ${ }^{20,21}$ These individual examples can be generalised in a simple 'rule-of-thumb' model, in which activation barriers decrease by $\sim 10 \mathrm{kcal} \mathrm{mol}^{-1}$ per three-membered ring fused to the breaking bond (corresponding to a $\sim 10^{7}$ fold rate enhancement at $298 \mathrm{~K}$ ). This leads to a 'hierarchy of delocalisation' that enables the quantitative prediction of strain-driven and delocalisation-enabled reactivity. 


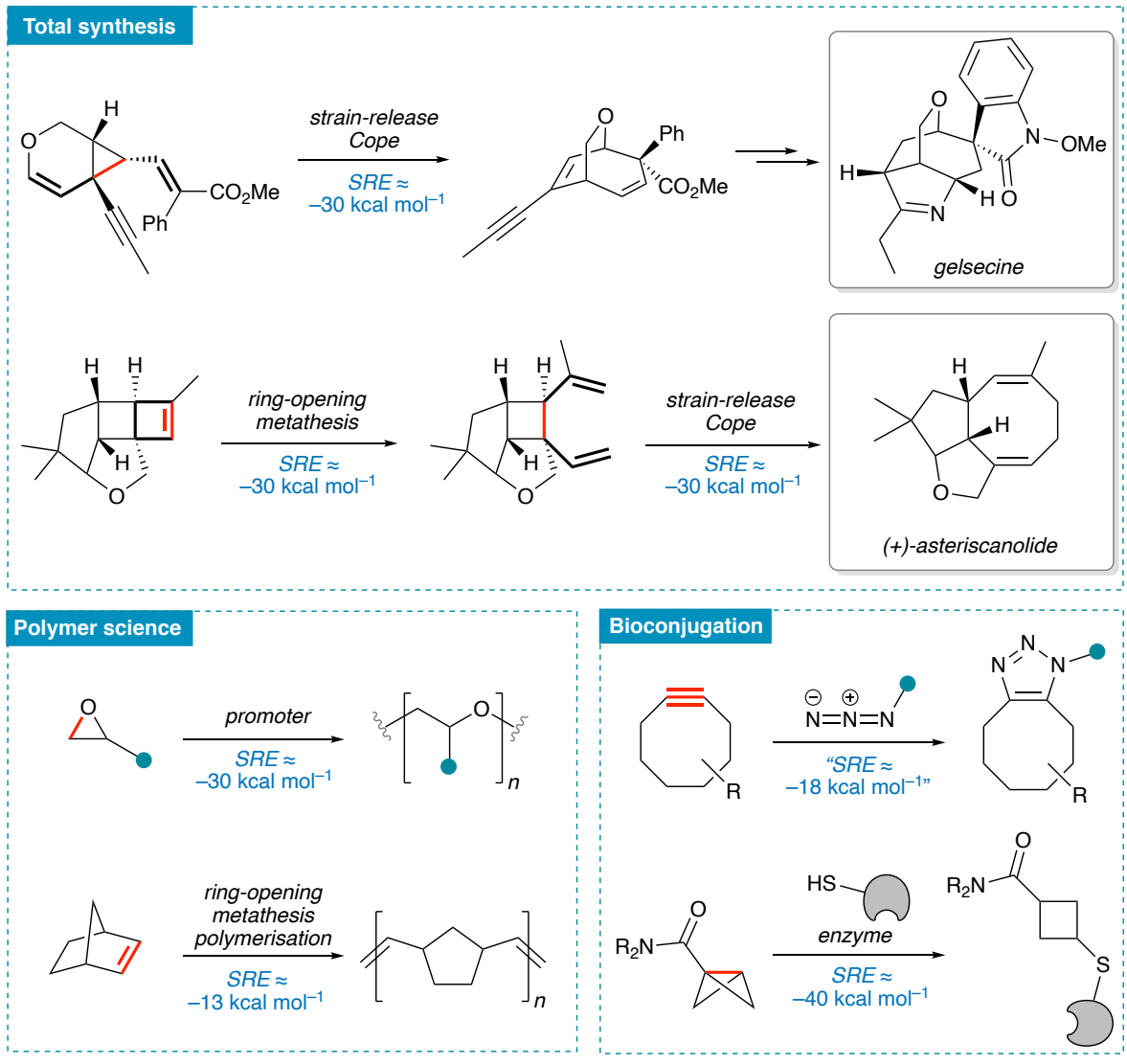

\section{Medicinal chemistry}

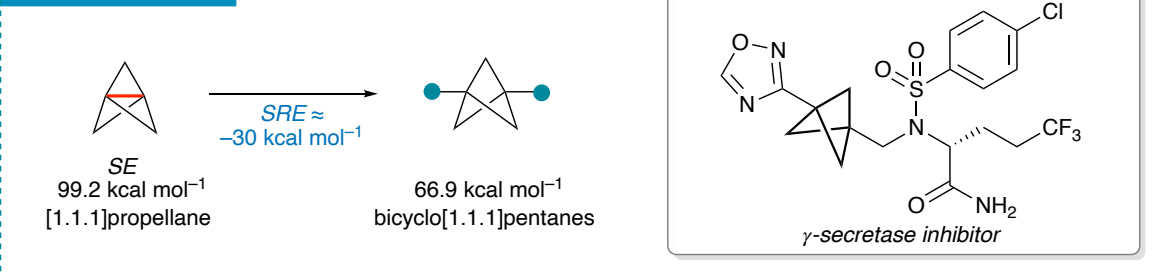

b) Delocalisation in small rings

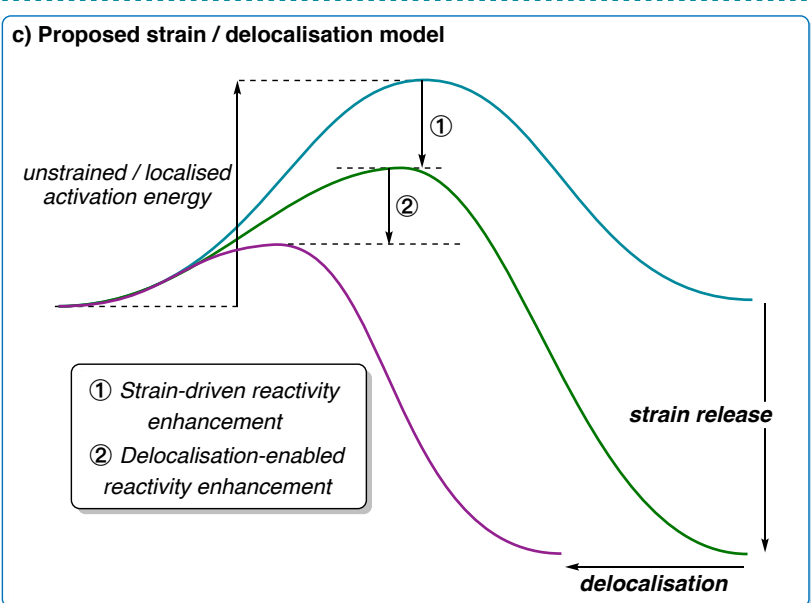

Fig. 1 a Examples of strain release reactivity across organic chemistry, with contexts including total synthesis, ${ }^{22,23}$ bioorthogonal conjugation reactions, ${ }^{6,7}$ ring-opening polymerisations, and bioisostere synthesis. ${ }^{24,25}$ b Delocalisation in three-membered rings introduced through Coulson \& Moffitt's bent bonding model, ${ }^{16}$ Dewar's $\sigma$-aromaticity ${ }^{17}$ and Weinhold and Landis' germinal hyperconjugation. ${ }^{18} \mathbf{c}$ This work: Strain release and delocalisation collectively enhance reactivity through lower, earlier activation barriers. 
Quantification of strain and delocalisation effects on reactivity. The effect of strain energy on reactivity can be characterised considering a linear relationship between the thermodynamic driving force of a reaction, $E_{r}$, with its activation barrier, $E_{a}$. An early example is the BellEvans-Polanyi (BEP) principle (Eq. 1, Fig. 2a), ${ }^{26,27}$ in which the difference in activation barrier for two similar reactions $\left(\Delta \mathrm{E}_{\mathrm{a}}\right)$ is proportional to the difference in driving force $\left(\Delta \mathrm{E}_{\mathrm{r}}\right)$. An analogous expression can be obtained from Marcus theory, originally developed to predict rates of electron transfer (Eq. 2) and later adapted for chemical reactions, where $E_{a, \text { int }}$ is the activation barrier in the absence of a driving force (i.e., $\Delta E_{r}=0$ ). ${ }^{28,29}$

$$
\begin{gathered}
\Delta E_{a}=\alpha \Delta E_{r} \\
E_{a}=E_{a, i n t}+\frac{1}{2} \Delta E_{r}+\frac{\Delta E_{r}^{2}}{16 E_{a, i n t}}
\end{gathered}
$$

In the context of the reactions of strained rings, both the BEP principle and Marcus theory predict that an increase in 'strain release energy' (SRE) should lead to a lower activation barrier. Based on these models, the similar strain energies of cyclopropane and cyclobutane would erroneously imply similar reaction profiles. This inability to correctly predict relative reactivities arises from the assumption that the $\mathrm{C}-\mathrm{C}$ bonds being broken are equivalent, despite being more delocalised in cyclopropane than in cyclobutane (vide supra); a more delocalised bond would be expected to have a lower intrinsic activation barrier as redistribution / unpairing of the bonding electrons incurs a lower energetic penalty. Activation barriers for a series of similar substrates should then depend on both strain release (through differences in $E_{r}$ ) and delocalisation (through differences in $E_{a, \text { int }}$ ). 

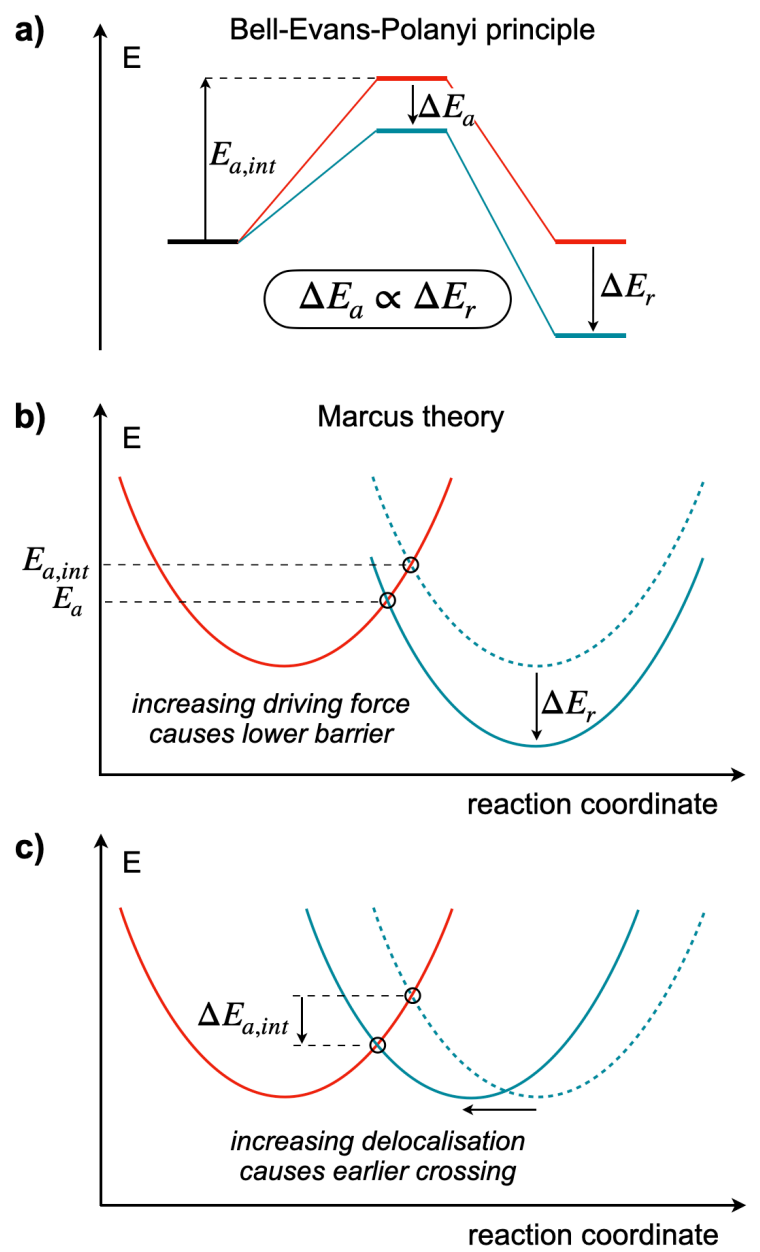

Fig. 2 a The Bell-Evans-Polanyi principle suggests a linear relationship between the reaction driving force $\left(\Delta \mathrm{E}_{\mathrm{r}}\right)$ and the increase in activation energy relative to the intrinsic activation barrier $\left(\Delta \mathrm{E}_{\mathrm{a}}\right)$. $\mathbf{b}$ Marcus theory describes the potential energy surface of a reaction as overlapping parabolas describing the reactants (red) and products (blue); their intersection point approximates the energy of the TS barrier. c Within Marcus theory, increasing the delocalisation of a given bond causes earlier curve crossing, decreasing the intrinsic activation barrier for a given reaction.

Radical addition to small-ring hydrocarbons. To explore the importance of delocalisation on reactivity, we selected a set of 12 acyclic, monocyclic and fused polycyclic hydrocarbons with ring sizes varying from three to five (Fig. 3a). Activation and reaction enthalpies $\left(\Delta \mathrm{H}^{\ddagger}\right.$ and $\Delta \mathrm{H}_{\mathrm{r}}$ ) were calculated at $298.15 \mathrm{~K}$ for the addition of a methyl radical to the bonds highlighted in red. ${ }^{30}$ Application of the BEP principle to this set of reactions revealed a poor correlation $\left(\mathrm{R}^{2}\right.$ $=0.51$ ) between $\Delta \mathrm{H}^{\ddagger}$ and $\Delta \mathrm{H}_{\mathrm{r}}$ (Fig. 3b), and a root mean squared error (RMSE) of $10.1 \mathrm{kcal}$ $\mathrm{mol}^{-1}$; particularly notable are the very similar reaction enthalpies for [1.1.1]propellane, cyclopropane and cyclobutane $\left(\Delta \mathrm{H}_{\mathrm{r}}=-28.2,-28.4\right.$ and $-26.8 \mathrm{kcal} \mathrm{mol}^{-1}$ respectively $)$ in spite of a $>30 \mathrm{kcal} \mathrm{mol}^{-1}$ span in activation enthalpies $\left(\Delta \mathrm{H}^{\ddagger}=5.0,26.4\right.$ and $36.1 \mathrm{kcal} \mathrm{mol}^{-1}$ respectively). A similarly poor correlation is found using Marcus theory (Fig. S1), as also observed by Hoz and co-workers. ${ }^{31,32}$ 
a) Test set

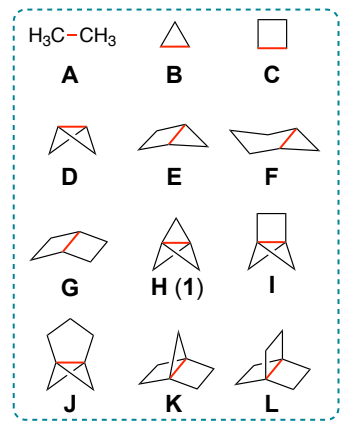

b)

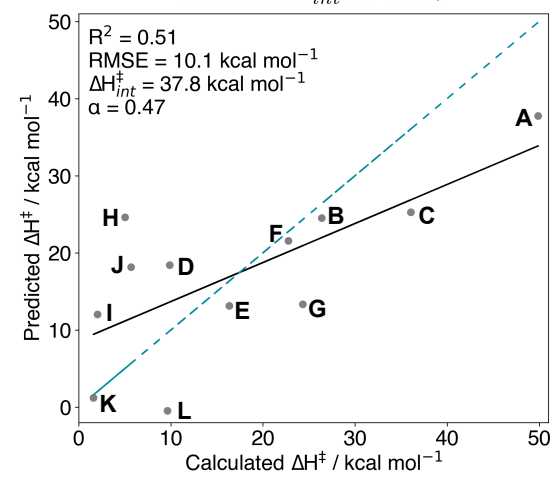

c) $\begin{gathered}\Delta H^{\ddagger}=\Delta H_{i n t}^{\ddagger}+\alpha \Delta H_{r}+\beta\left(2-N_{\text {occ }}\right) \\ \mathrm{R}^{2}=0.97\end{gathered}$

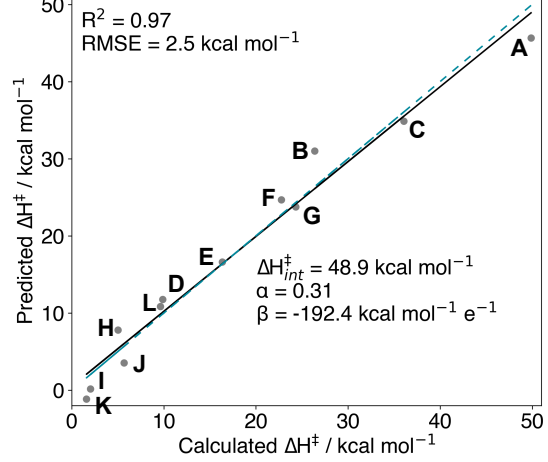

d) Hierarchy of delocalisation

Transition state geometries

$\left[\mathrm{A}+\mathrm{CH}_{3} \cdot\right]^{\ddagger}$

$\Delta \mathrm{H}^{\ddagger}=49.9$

$\Delta \mathrm{H}_{\mathrm{r}}=0.0$

$2-N_{\text {occ }}=0.017$

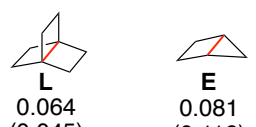

$\begin{array}{ll}0.064 & 0.081 \\ (0.045) & (0.116)\end{array}$

\begin{tabular}{c}
$\left(\begin{array}{c}0.116) \\
\perp\end{array}\right.$ \\
\hline
\end{tabular}

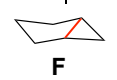

0.069

$(0.099)$

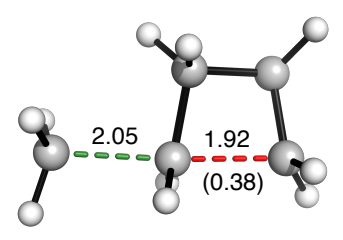

$\left[\mathrm{C}+\mathrm{CH}_{3}\right]^{\ddagger}$

$\Delta \mathrm{H}^{\ddagger}=36.1$

$\Delta \mathrm{H}_{\mathrm{r}}=-26.8$

$2-N_{\text {occ }}=0.029$

\section{delocalisation}

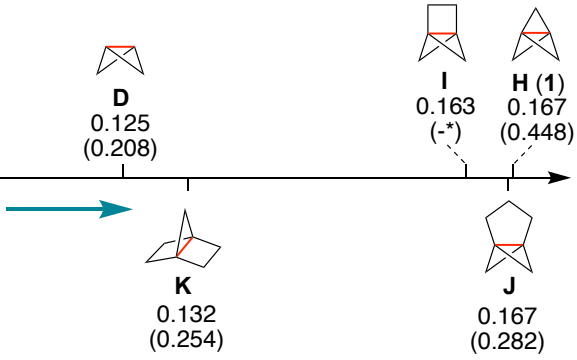

$(0.254)$

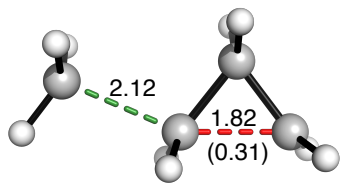

$\left[\mathrm{B}+\mathrm{CH}_{3}\right]^{\ddagger}$

$\Delta \mathrm{H}^{\ddagger}=26.4$

$\Delta \mathrm{H}_{\mathrm{r}}=-28.4$

$2-N_{\text {occ }}=0.046$

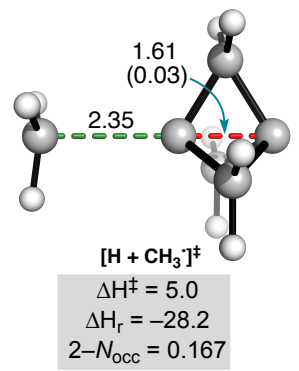

Electron density difference plots at each transition state geometry

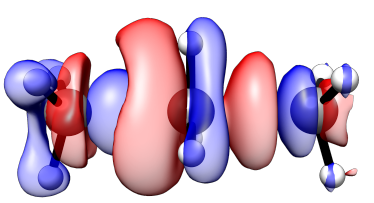

$\left.\rho^{\ddagger}{ }_{[\mathbf{A}}+\mathrm{CH} 3\right]-\left(\rho_{\mathbf{A}}+\rho_{\mathrm{CH} 3}\right)$

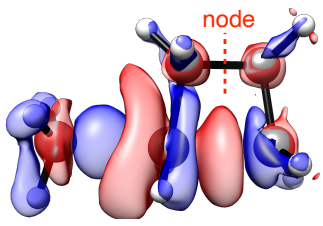

$\rho^{\ddagger}[\mathrm{C}+\mathrm{CH} 3]-\left(\rho_{\mathrm{C}}+\rho_{\mathrm{CH} 3}\right)$

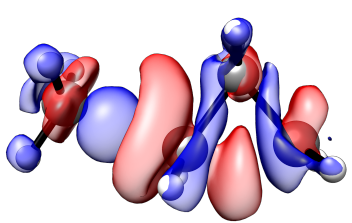

$\left.\rho^{\ddagger}{ }_{[\mathrm{B}}+\mathrm{CH} 3\right]-\left(\rho_{\mathrm{B}}+\rho_{\mathrm{CH} 3}\right)$

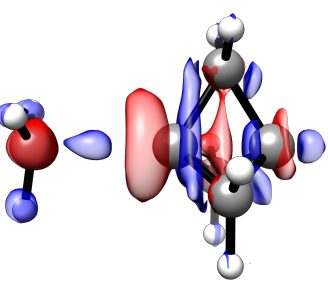

$\left.\rho^{\ddagger}{ }_{[\mathrm{H}}+\mathrm{CH} 3\right]-\left(\rho_{\mathrm{H}}+\rho_{\mathrm{CH} 3}\right)$

$\Delta \rho>0$ (density accumulation)

$\Delta \rho<0$ (density depletion)

Fig. 3 a Set of acyclic, monocyclic, and fused polycyclic hydrocarbons used for BEP plots. b BEP plot $\left(\Delta \mathrm{H}_{\mathrm{r}}\right.$ vs $\left.\Delta \mathrm{H}^{\ddagger}, \mathrm{kcal} \mathrm{mol}^{-1}\right)$ for the addition of a methyl radical to the red bonds of molecules defined in $\mathbf{a}$. c Prediction of $\Delta \mathrm{H}^{\ddagger}$ from $\Delta \mathrm{H}_{\mathrm{r}}$ and $2-N_{\text {occ }}$ (Eq. 3); $\alpha$ and $\beta$ are optimised coefficients for $\Delta \mathrm{H}_{\mathrm{r}}$ and $2-N_{\text {occ }}$, respectively. The blue dashed line denotes perfect correlation. $\mathbf{d}$ Trend in delocalisation of each red bond in a, through either 2-Nocc or (1-ELF, in parentheses). *No bond critical point located for [2.1.1]propellane. e Selected TS geometries (distances in $\AA$ ), enthalpies $\left(\mathrm{kcal} \mathrm{mol}^{-1}\right), 2-N_{\text {occ }}$ values $(e)$ and electron density difference plots (isovalue of $0.015 \mathrm{e}^{-3}$ ) for the addition of a methyl radical to ethane, cyclobutane, cyclopropane and [1.1.1]propellane. Difference between TS and equilibrium bond lengths shown in parentheses.

To test the hypothesis that delocalisation accounts for the discrepancy between strain and reactivity for these systems, we calculated the occupation number $\left(N_{o c c}\right)$ of the natural bond 
orbital (NBO) corresponding to the breaking bond, where deviation from a full occupation of 2 (denoted 2-Nocc) describes the degree of delocalisation - in particular that proposed to occur in three-membered rings. Increased donation from the $\sigma$ bond to be broken into a geminal $\sigma^{*}$ orbital will increase the value of $2-N_{\text {occ }}$, capturing the geminal hyperconjugation (delocalisation) effect proposed by Weinhold and Landis (Fig. 1b). Incorporation of this $2-N_{\text {occ }}$ parameter into the BEP model using multiple linear regression (Eq. 3 and Fig. 3c) resulted in an excellent correlation $\left(\mathrm{R}^{2}=0.97\right)$ and low $\mathrm{RMSE}\left(2.5 \mathrm{kcal} \mathrm{mol}^{-1}\right)$.

$$
\Delta H^{\ddagger}=\Delta H_{i n t}^{\ddagger}+\alpha \Delta H_{r}+\beta\left(2-N_{o c c}\right)
$$

The negative value of the delocalisation parameter $\beta\left(-192 \mathrm{kcal} \mathrm{mol}^{-1} \mathrm{e}^{-1}\right)$ reflects the decrease in the intrinsic barrier due to delocalisation, while the increasing value of $2-N_{\text {occ }}$ in the series cyclobutane (0.029), cyclopropane (0.046), bicyclo[1.1.0]butane (0.125) and [1.1.1]propellane (0.167) shows that the reacting bond becomes more delocalised, and therefore more susceptible to cleavage. Inclusion of the $2-N_{\text {occ }}$ parameter into the Marcus equation leads to nearly identical results (Fig. S2). A hierarchy of bond delocalisation can be constructed to rank different molecules by the extent of delocalisation, therefore intrinsic reactivity (Fig. 3d). Molecules further to the right will have smaller intrinsic activation barriers, distinct from barrier lowering due to the thermodynamics of bond cleavage. The values of $2-N_{\text {occ }}$ were found to be in good agreement with an alternative density-based delocalisation parameter, 1-ELF, where ELF is the electron localisation function at the bond critical point. ${ }^{33}$ This latter parameter provides a similarly strong correlation and low error $\left(R^{2}=0.94, \mathrm{RMSE}=3.3 \mathrm{kcal} \mathrm{mol}^{-1}\right.$, Fig. S3)

To further probe the role of delocalisation in defining the reactivity of small rings, we employed electron density difference plots (EDDPs) to examine the rearrangement of electron density at the TS for ethane, cyclobutane, cyclopropane and [1.1.1]propellane (Fig. 3e). EDDPs are constructed by subtracting the electron density of each distorted fragment (e.g., $\mathrm{CH}_{3}{ }^{\cdot}$ and ethane) from the total TS electron density, and gives an indication of the flow of electron density during the reaction: blue lobes indicate density accumulation, and red lobes indicate depletion. For the reaction of the localised $\mathrm{C}-\mathrm{C}$ bond in ethane, the EDDP involves the expected removal of electron density from the breaking $\mathrm{C}-\mathrm{C}$ bond, and accumulation in the forming $\mathrm{C}-$ $\mathrm{C}$ bond, with some accumulation onto the hydrogen atoms of the central $\mathrm{CH}_{3}$, presumably due to rehybridization upon planarisation. Cyclobutane exhibits a similar picture, with a node between the bridging $\mathrm{CH}_{2}$ units indicating a lack of communication through-bond. However, at the cyclopropane TS, lateral accumulation of density between the bridging methylene and the central / leaving methylene groups indicates disturbance of through-bond delocalisation- in agreement with Coulson and Moffitt's original proposal that the electron density of the 
cyclopropane $\mathrm{C}-\mathrm{C}$ bonds is delocalised, and Houk's OITB model in which the $\mathrm{C}-\mathrm{C} \sigma$ bond is stabilised by the $\mathrm{C}-\mathrm{C} \sigma^{*} \mathrm{MOs}$ to the methylene bridge. [1.1.1]Propellane shows an equivalent effect to cyclopropane, however the delocalisation now involves three methylene groups instead of one. ${ }^{19}$

We next investigated whether the number of three-membered rings fused to the breaking bond alone could be used as a metric for delocalisation ( $n_{3}$, Eq. 4$)$.

$$
\Delta H^{\ddagger}=\Delta H_{i n t}^{\ddagger}+\alpha \Delta H_{r}+\beta n_{3}
$$

Using this parameter in place of $\left(2-N_{\text {occ }}\right)$ leads to a remarkably accurate predictor of reactivity (Fig. 4a): for each three-membered ring fused to the breaking $\mathrm{C}-\mathrm{C}$ bond, the intrinsic activation energy is lowered by $\sim 10 \mathrm{kcal} \mathrm{mol}^{-1}$, corresponding to a $\sim 10^{7}$-fold increase in the rate constant at $298 \mathrm{~K}$. This simple model therefore not only explains the fundamentally greater reactivity of cyclopropane over cyclobutane, but also the contrasting reactivites of [1.1.1]propellane and cyclopropane, where the greatly enhanced reactivity of the former can be attributed entirely to the higher number of three-membered rings fused to the breaking bond $\left(n_{3}\right.$ $=3$ ), which maximises delocalisation effects. We further predict a hierarchy of reactivity for the series: bicyclo[1.1.0]butane > bicyclo[2.1.0]pentane > bicyclo[2.2.0]hexane $\left(\Delta \mathrm{H}^{\ddagger}=9.9\right.$, 16.4 and $24.3 \mathrm{kcal} \mathrm{mol}^{-1}$ ), where the activation energy contrasts with their respective reaction enthalpies $\left(\Delta \mathrm{H}_{\mathrm{r}}=-41.5,-52.9\right.$ and $\left.-52.4 \mathrm{kcal} \mathrm{mol}^{-1}\right)$. Our model, based on the number of three-membered rings appended to the breaking bond, quantitively accounts for this trend, delivering predicted $\Delta \mathrm{H}^{\ddagger}$ values of $9.0,14.0$ and $24.7 \mathrm{kcal} \mathrm{mol}^{-1}$, respectively. In summary, variation of the number of three-membered rings fused to a breaking bond is a simple and predictable way to modulate the reactivity of the system, for example switching the behaviour of a molecule from a highly-reactive bioconjugate warhead (e.g., bicyclo[1.1.0]butanes derived from $\mathbf{D})^{7,34}$ to an inert lipid tail group (e.g. bicyclo[2.2.0]hexane-derived 'ladderanes' based upon $\mathbf{G}){ }^{35}$ 
a) $\mathrm{CH}_{3} \cdot$ addition

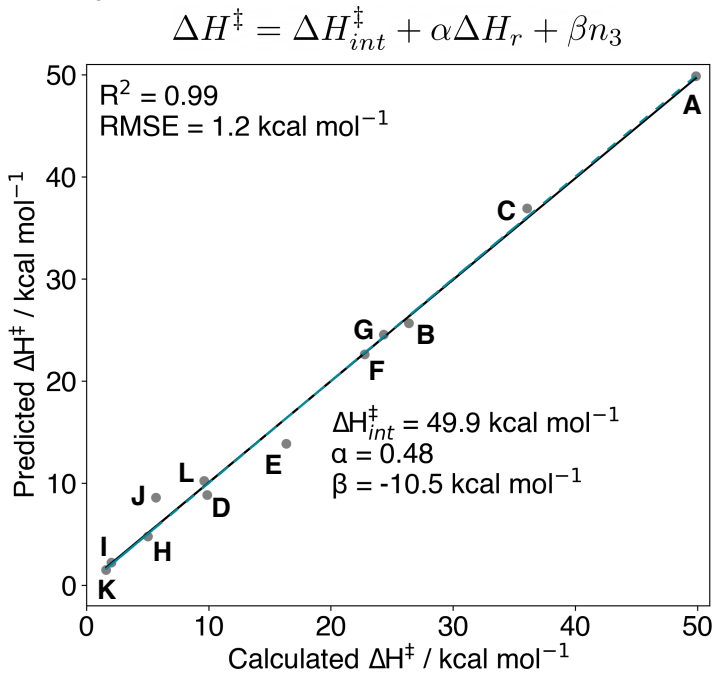

b) $\mathrm{NH}_{2}^{-}$addition

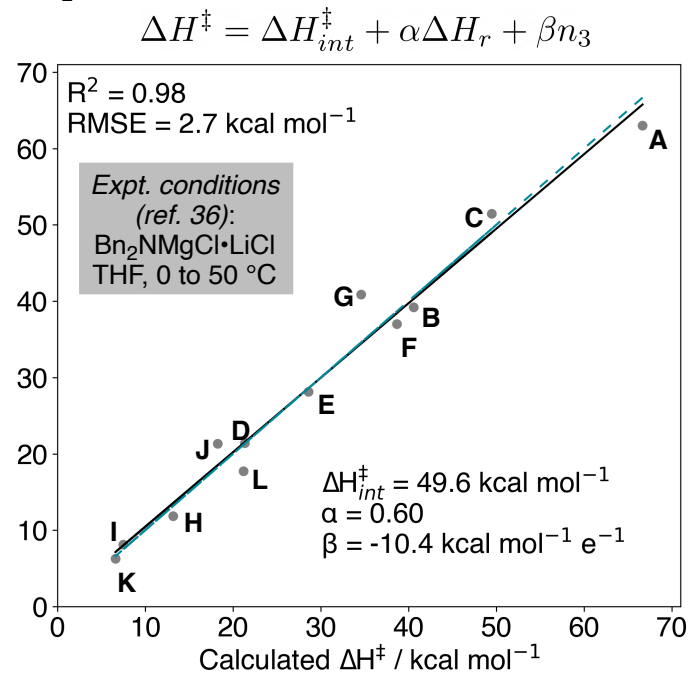

c) Strain-release amination (ref 36)
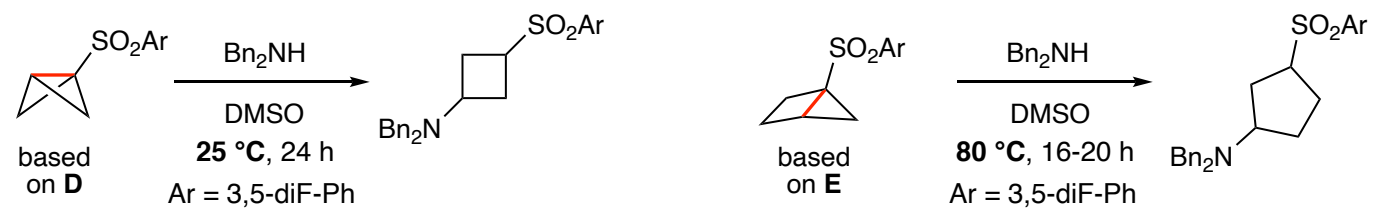

d) Heterocyclic reactivity
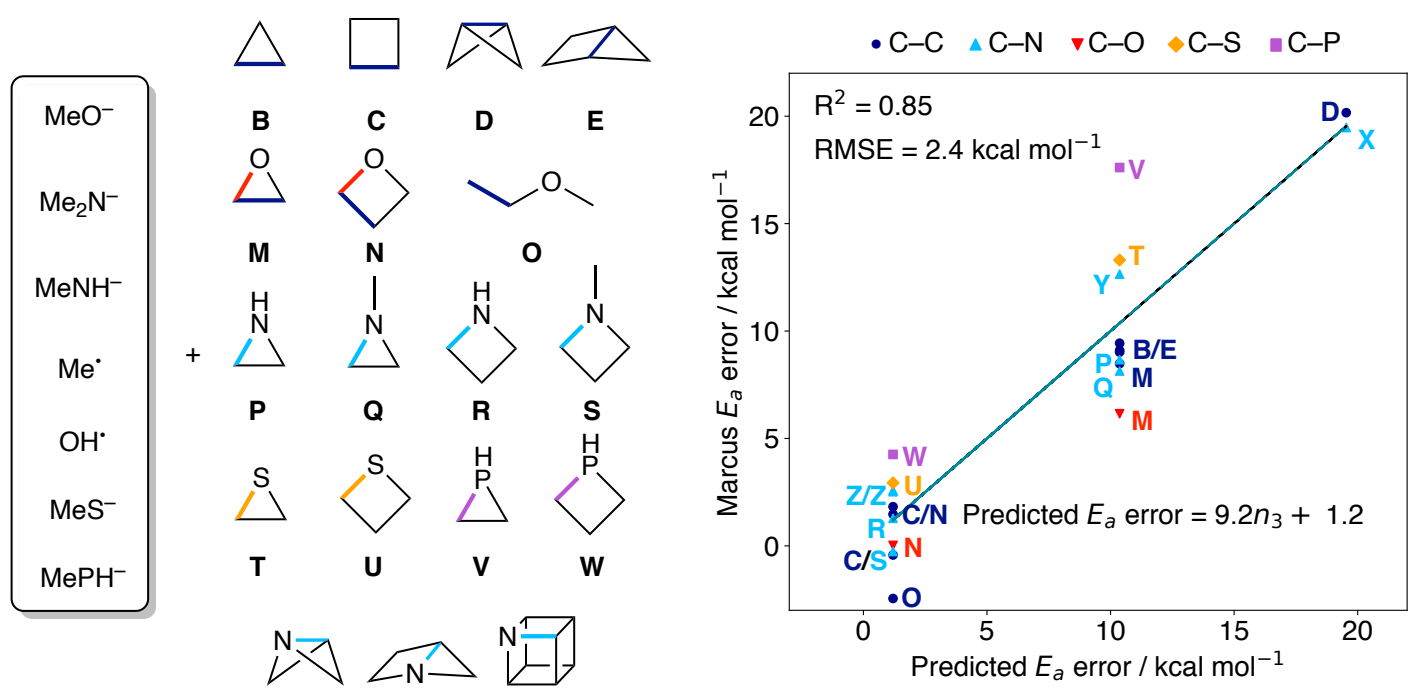

Fig. 4 a MLR plots for the prediction of $\Delta \mathrm{H}^{\ddagger}$ from $\Delta \mathrm{H}_{\mathrm{r}}$ and $n_{3}$ for the hydrocarbon set with $\mathrm{CH}_{3}{ }^{\circ}$ (a) and $\mathrm{NH}_{2}{ }^{-}$(b) using Eq. 5. $\alpha$ and $\beta$ are optimised coefficients for $\Delta \mathrm{H}_{\mathrm{r}}$ and $n_{3}$ respectively. The blue dashed lines denote perfect correlation. c Anionic and radical additions to a set of 18 heterocyclic molecules, and the error in activation energy $\left(E_{a}\right)$ predicted by Marcus theory vs $2-N_{\text {occ. }}$. Reaction data from Refs. 31 and ${ }^{32}$.

Strain-release amination. The generalised delocalisation model in Eq. 4 is also applicable to anionic processes, such as the addition reactions of amide anions to $\mathbf{D}, \mathbf{E}$ and $\mathbf{H}$ (Fig. 3a) developed by Baran and co-workers. ${ }^{34,36}$ Using $\mathrm{NH}_{2}{ }^{-}$as a model nucleophile, excellent correlation $\left(R^{2}=0.98\right)$ was obtained between activation and reaction enthalpies (Fig. 4b). A similar 
$\beta$ coefficient of $\sim-10 \mathrm{kcal} \mathrm{mol}^{-1}$ was obtained, suggesting that the effect of delocalisation on reaction barriers may be independent of reaction type. Notably, if delocalisation effects were removed from [1.1.1]propellane, its activation barrier for this reaction would increase by $\sim 30$ $\mathrm{kcal} \mathrm{mol}^{-1}$, rendering it inert under the reaction conditions. In other words, the release of strain energy alone is insufficient to enable the observed reaction - instead, delocalisation causes an earlier, lower energy TS, leading to its 'spring-loaded' behaviour. The same is true of bicyclo[1.1.0]butanes (D) and bicyclo[2.1.0]pentanes (E), where activation barriers would increase by $\sim 20$ and $\sim 10 \mathrm{kcal} \mathrm{mol}^{-1}$ respectively, in the absence of delocalisation. This effect is corroborated by experiments on the addition of dibenzylamine to bicyclo[1.1.0]butane and bicyclo[2.1.0]pentane sulfones (Fig. 4c), where the former affords the cyclobutyl amine product at ambient temperature, whereas the latter requires heating to $80^{\circ} \mathrm{C}$ to form the equivalent cyclopentane. ${ }^{36}$ This reactivity difference directly opposes the expected behaviour from SREs alone $\left(-40.2\right.$ and $-48.1 \mathrm{kcal} \mathrm{mol}^{-1}$ for bicyclo[1.1.0]butane and bicyclo[2.1.0]pentane, respectively).

Heteroatom effects on delocalisation and reactivity. To investigate whether delocalisation also plays a role in the reactivity difference between hetereosubstituted cyclopropanes and their four-membered homologues, we analysed a dataset of anionic and radical ring-opening reactions of $C, N, O, P$ and S-substituted heterocycles (Fig. $4 \mathrm{~d}$ and Fig. S4). ${ }^{31,32}$ This set was generated by Hoz and co-workers, who observed a large error between the calculated and predicted barriers ( $\left.E_{a}\right)$ from the Marcus equation (Eq. 2). We found that this error again correlates reasonably well with the number of three-membered rings $\left(n_{3}, \mathrm{R}^{2}=0.85\right)$, indicating that delocalisation effects are once more primarily responsible for reactivity differences between three- and four-membered rings. Interestingly, similarity between $\mathrm{C}-\mathrm{N}$ and $\mathrm{C}-\mathrm{C}$ errors indicates that aza- and carbocycles experience a similar barrier-lowering contribution from delocalisation effects. In contrast, oxacycles exhibit a slightly diminished contribution, and thirdrow elements display a marked enhancement. This pattern is likely explained by electronegativity differences, where diminishing effective nuclear charge increases the propensity for delocalisation. Additionally, while factors such as dipolar interactions - expected to be important for activation barriers involving heteronuclear bond cleavage - are also missing from this simple model, as before the number of three-membered rings fused to the breaking bond explains the variance in the intrinsic activation barrier. In this case, it is possible that these polar effects are similar between three- and four-membered rings of the same type, therefore approximately cancel when estimating reactivity differences.

Rule of thumb for reactivity prediction. The delocalisation effects that explain the enhanced reactivity of three-membered rings can be simplified to a 'rule of thumb' model that rapidly 
estimates relative reactivity. This model employs the modified BEP approach (Eq. 5) and tabulated SREs that are available for most common substrates; $\alpha$ is taken as 0.5 and $\beta$ as 10 $\mathrm{kcal} \mathrm{mol}{ }^{-1}$ based on the results obtained above. Despite the intrinsic activation barrier $\left(\Delta \mathrm{H}^{\ddagger}{ }_{\text {int }}\right)$ being unknown, the difference in activation barriers between two substrates $\left(\Delta \Delta \mathrm{H}^{\ddagger}\right)$ can be estimated as follows:

$$
\Delta \Delta H^{\ddagger} \approx 0.5 \Delta S R E-10 \Delta n_{3}
$$

We applied this model to rationalise the different reactivity for the radical addition reactions of [1.1.1]propellane $\mathbf{H}$, bicyclo[1.1.0]butane $\mathbf{D}$ and housane $\mathbf{E}$ (bicyclo[2.1.0]pentane) with $\mathrm{BrCCl}_{3}$ and $\mathrm{CCl}_{4}$ (Fig. 5a). ${ }^{37,38}$ These reactions were first reported by Wiberg and co-workers, who found that while [1.1.1]propellane and bicyclo[1.1.0]butane readily undergo addition, housane showed no reactivity under these conditions. Additionally, competition reactions showed that [1.1.1]propellane undergoes significantly more rapid reaction than bicyclo[1.1.0]butane. Wiberg noted that the observed reactivity of these three molecules directly opposed the trend in SRE (Fig. 5a), and that properties such as the ionisation potential and local charge concentration were also unable to even qualitatively explain the reactivity trend. In contrast, our model correctly predicts the observed trend, with estimated activation barriers relative to [1.1.1]propellane of 4.0 and $10.1 \mathrm{kcal} \mathrm{mol}^{-1}$ for bicyclo[1.1.0]butane and housane respectively (Fig. 5b). To test the accuracy of these predictions, activation and reaction enthalpies were calculated for the addition of $\mathrm{CCl}_{3}{ }^{\cdot}$ to each of these small rings. The lowest barrier was calculated for [1.1.1]propellane $\left(\Delta \mathrm{H}^{\ddagger}=0.5 \mathrm{kcal} \mathrm{mol}^{-1}\right)$, with addition barriers for bicyclo[1.1.0]butane and housane greater by 3.5 and $10.2 \mathrm{kcal} \mathrm{mol}{ }^{-1}$, respectively. ${ }^{39}$ Considering the simplicity of the 'rule of thumb' model, the numerical accuracy is certainly acceptable.

General use of the strain / delocalisation model. We have constructed a set of SREs and $2-N_{\text {occ }}$ values for each bond in a range of commonly-employed small ring-containing molecules to permit the easy application of our strain / delocalisation model and explore the generalisability of our findings (Fig. 5c). SREs (DLPNO-CCSD(T)/def2-QZVPP//B2PLYP$\mathrm{D} 3 \mathrm{BJ} /$ def2-TZVP) were estimated by constructing balanced hydrogenation reactions, by analogy to homodesmotic reactions, in which the numbers of each bond type (e.g., $\mathrm{C}-\mathrm{C}, \mathrm{C}=\mathrm{C}$ ) and atom type (e.g., $\mathrm{C}\left(\mathrm{H}_{3}\right)(\mathrm{C}), \mathrm{C}\left(\mathrm{H}_{2}\right)\left(\mathrm{C}_{2}\right), \mathrm{C}(\mathrm{H})\left(\mathrm{C}_{3}\right)$ ) are equal in the strained reactants and 'unstrained' products. An example calculation is illustrated in Fig. $5 \mathrm{c}$, with the complete set listed in Figs. S5-11. Where available, we have compared our SRE values to those calculated or measured previously (Table S1/S2), ${ }^{2,11}$ with which we generally find close agreement. Alongside the fused small ring systems shown in Fig. 3a, we have also included data for 

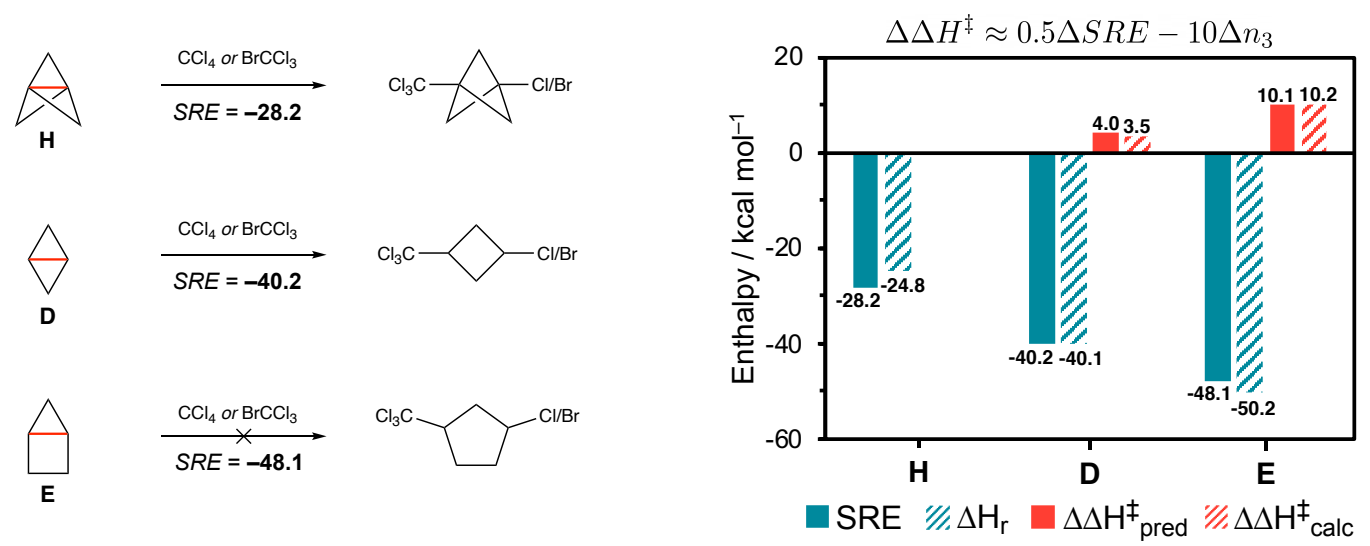

c) Strain release energy and (2- $\left.N_{\text {occ }}\right)$

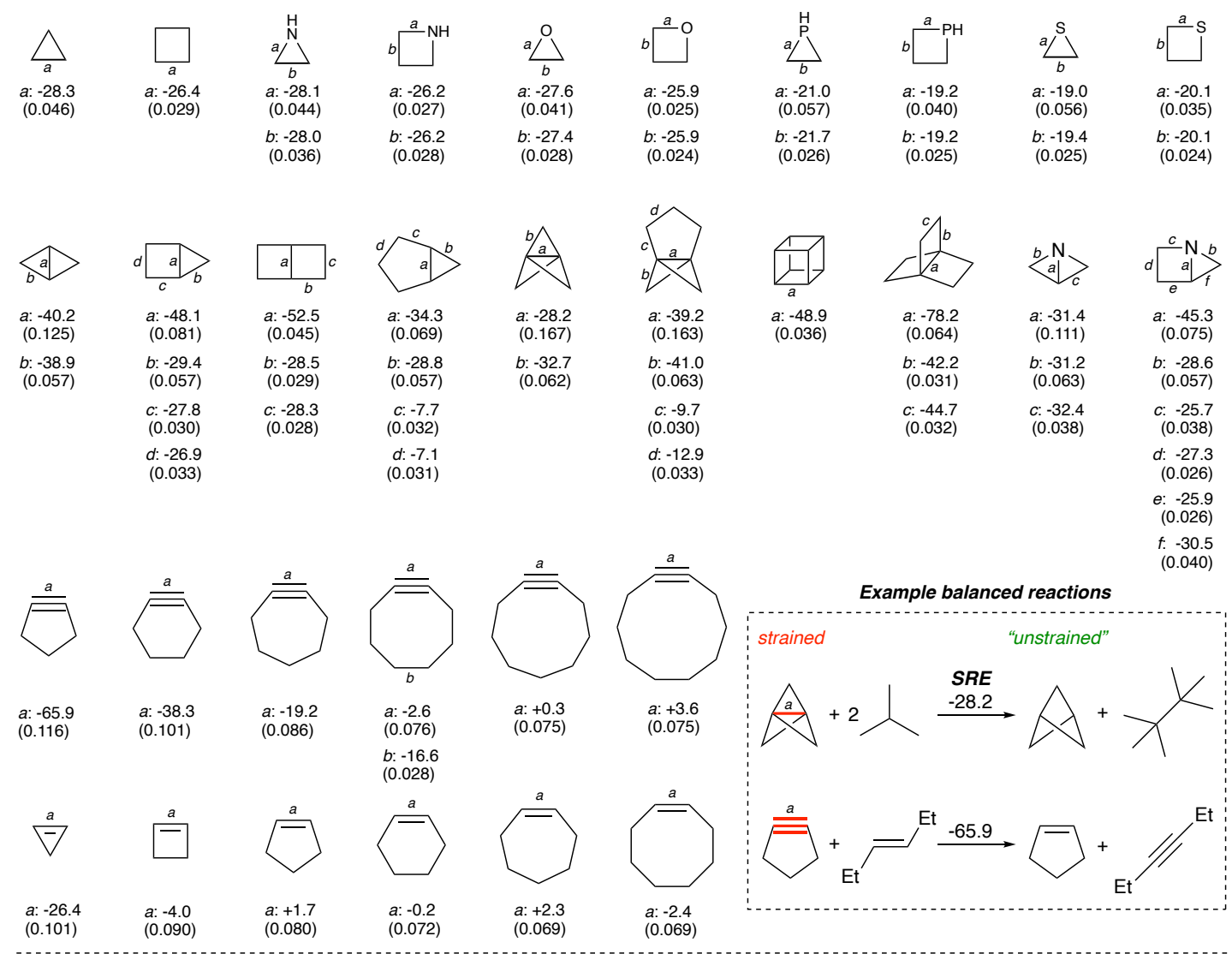

d) 'Strain-release' [3 + 2] cycloaddition
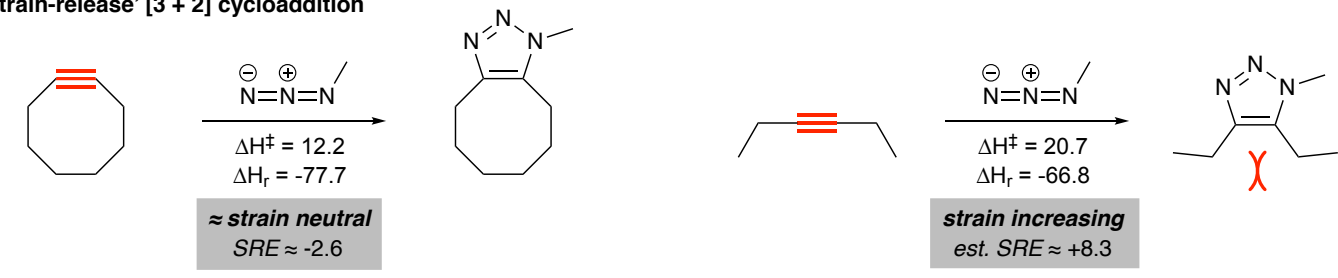

Fig. 5. a $\mathrm{CCl}_{4}$ and $\mathrm{BrCCl}_{3}$ react rapidly with [1.1.1]propellane (H) and bicyclo[1.1.0]butane (D), yet housane $(E)$ is inert under the same conditions. b Predicted relative activation enthalpies $\left(\Delta \Delta \mathrm{H}^{\ddagger}\right.$ pred $)$ based on SRE using Eq. 5, and calculated activation enthalpies $\left(\Delta \Delta \mathrm{H}_{\text {calc }}\right)$ for comparison. $\Delta \mathrm{H}_{\mathrm{r}}$ is included for comparison with SRE. All enthalpies in kcal mol-1. c Set of strain release energies (SREs, $\mathrm{kcal} \mathrm{mol}^{-1}$ ) and 2-Nocc values (e) per bond type for a range of mono-, bi- and tricyclic ring systems, cyclic alkynes and alkenes. $\mathbf{d}$ Activation and reaction enthalpies $\left(\mathrm{kcal} \mathrm{mol}^{-1}\right)$ for [3 +2$]$ cycloadditions between methyl azide and cyclooctyne or hex-3-yne. 
cycloalkynes and cycloalkenes given their wide applications in strain release chemistry. ${ }^{40-43}$ While a small ring is not opened in the latter two classes, we anticipate the same principles of our strain release / delocalisation model will apply: the electrons in a $\pi$ bond are less strongly paired than those in an equivalent $\sigma$ bond due to poorer orbital overlap. As a result, the $\pi$ bond will inherently be more reactive because the electrons may unpair more easily at an earlier point on the reaction coordinate, resulting in a lower intrinsic activation energy - distinct from the effect of strain release. For the cycloalkene series, both SRE and $2-N_{\text {occ }}$ increase as the size of the ring decreases, and as a result we expect delocalisation to enhance the strain release effect accompanying $\pi$ bond cleavage. Experimentally, we anticipate this phenomenon to be manifested in, for example, the rate of [2 + 2] cycloadditions in ring-opening metathesis reactions, where cyclobutenes will react faster than cyclopentenes not only because of the strain release effect, but because of increased bond delocalisation.

For cycloalkynes, the distinction between strain-driven and delocalisation-enabled reactivity is exemplified by a comparison of reactivity of the $\pi$ and $\sigma$ bonds of cyclooctene (Fig. 5c): the SREs ( -2.6 and $-16.6 \mathrm{kcal} \mathrm{mol}^{-1}$ for ' $a$ ' and ' $b$ ', respectively) would erroneously predict greater reactivity of the $\sigma$ bond, despite the well-established [3 + 2] azide-alkyne cycloaddition chemistry pointing to the opposite result. ${ }^{42,43}$ However, the $2-N_{\text {occ }}$ values ( 0.076 and 0.028 for ' $a$ ' and ' $b$ ', respectively) recover the expected reactivity pattern - the more delocalised $\pi$ bond will have a lower intrinsic activation barrier, and therefore reacts faster despite the lack of strain release bias. We were particularly surprised to see such a small SRE $\left(-2.6 \mathrm{kcal} \mathrm{mol}^{-1}\right)$ for the transformation from the strained alkyne to Z-cyclooctene (bond type ' $a$ ', Fig. 5c). This result leads to the conclusion that [3 + 2] azide-alkyne cycloadditions with 'unstrained' acyclic alkynes are net strain-increasing (Fig. 5d), perhaps because of increased steric clashing, or the sacrifice of two $C(s p)-C\left(s p^{3}\right)$ bonds for two weaker $C\left(s p^{2}\right)-C\left(s p^{3}\right)$ bonds. ${ }^{44}$ Bending the alkyne by its incorporation into the eight-membered ring counteracts this strain increase, an interpretation supported by the increasing positive sign of the SRE as the cycloalkyne ring size increases from 8 to 10 .

\section{Conclusion}

While strain energy is often invoked to rationalise trends in reactivity, it is often insufficient to explain observed reaction kinetics. This work proposes bond delocalisation as an equally important factor necessary to understand the observed 'spring-loaded' reactivity often associated with strain release in small rings, cycloalkynes and cycloalkenes. Evaluation of small-ring radical and anionic addition reactions in three-membered rings reveals that delocalisation effects operate in both carbo- and heterocyclic systems, and are critical for the success of these 
reactions. Increasing delocalisation also explains the enhanced reactivity of $\pi$ bonds vs $\sigma$ bonds, independently of differences in bond strengths. We anticipate that this new understanding of the reactivity of strained molecules will stimulate future developments in synthetic methodology, providing access to new molecules of relevance for organic synthesis, medicinal chemistry, polymer science and bioconjugation reactions.

\section{Methods}

Minima and TSs were initially identified using autodE ( $\mathrm{v} 1.0 .0 \mathrm{~b} 3),{ }^{45}$ with low energy conformers located using the ETKDGv2 algorithm implemented in RDKit v. 2019.03.4, ${ }^{46}$ and optimised using GFN2-xTB implemented in XTB ( $v$ 6.2.2) $)^{47}$ followed by PBE0-D3BJ/def2-TZVP//PBE0$\mathrm{D} 3 \mathrm{BJ} /$ def2-SVP ${ }^{48,49}$ in ORCA (v 4.2.1). ${ }^{50}$ Anionic reactions were run using the $\mathrm{GBSA}^{51}$ / $\mathrm{CPCM}^{52}$ solvent models for THF in XTB / ORCA, respectively. Geometries and energies were then refined in ORCA at the [DLPNO-CCSD(T)/def2-QZVPP (TightPNO)//B2PLYPD3BJ/def2-TZVP] level of theory $\left(\mathrm{CH}_{3} \cdot\right.$ reactions) or [SMD(THF)/DLPNO-CCSD(T)/ma-def2QZVPP (TightPNO)//SMD(THF)/B2PLYP-D3BJ/def2-TZVP (ma-def2-TZVP on N)] level of theory ( $\mathrm{NH}_{2}^{-}$reactions) ${ }^{48,53-55}$ All calculations used the resolution of the identity approximation (RIJCOSX), ${ }^{56}$ with the appropriate auxiliary basis sets ${ }^{57}$ and the Grid6/GridX6 combination of integration grids. NBO occupation numbers were calculated using the NBO program $(v 7.0)$, and density-based descriptors were calculated with Multiwfn ( $v 3.6) .{ }^{58}$ All data processing was carried out using the Scikit-learn package in Python $3.7,,^{59}$ and regression plots were generated with Matplotlib. ${ }^{60}$

\section{Data Availability}

A script to generate all linear regression data and plots discussed in this paper is available in the Supporting Information. Cartesian coordinates and energies of all stationary points are available as part of the Supporting Information.

\section{Acknowledgements}

We thank S. Zavitsanou and M. de la Puente for insightful discussions. A. J. S. thanks the EPSRC Centre for Doctoral Training in Synthesis for Biology and Medicine for a studentship (EP/L015838/1), generously supported by AstraZeneca, Diamond Light Source, Defence Science and Technology Laboratory, Evotec, GlaxoSmithKline, Janssen, Novartis, Pfizer, Syngenta, Takeda, UCB and Vertex. A. J. S. also thanks the Oxford-Radcliffe Scholarship for a studentship, and the EPSRC Doctoral Prize (EP/T517811/1) for support. This work used the Cirrus UK National Tier-2 HPC Service at EPCC (http://www.cirrus.ac.uk) funded by the University of Edinburgh and EPSRC (EP/P020267/1), and we thank the EPSRC Centre for Doctoral Training for Theory and Modelling in Chemical Sciences (EP/L015722/1) for providing access to the Dirac cluster at Oxford.

\section{Author contributions}

A.J.S., E.A.A. and F.D. conceptualised the study, analysed the data and wrote the manuscript. A.J.S. implemented the models and carried out the calculations. F.D., E.A.A. and R.C.S. supervised the study.

\section{Competing interests}

The authors declare no competing interests.

\section{References}

1. Clayden, J., Greeves, N. \& Warren, S. Organic Chemistry. (Oxford University Press, 2012). 
2. Wiberg, K. B. The Concept of Strain in Organic Chemistry. Angew. Chem. Int. Ed. Engl. 25, 312-322 (1986).

3. Ebner, C. \& Carreira, E. M. Cyclopropanation Strategies in Recent Total Syntheses. Chem. Rev. 117, 11651-11679 (2017).

4. Herzberger, J., Niederer, K., Pohlit, H., Seiwert, J., Worm, M., Wurm, F. R. \& Frey, H. Polymerization of ethylene oxide, propylene oxide, and other alkylene oxides: Synthesis, novel polymer architectures, and bioconjugation. Chem. Rev. 116, 2170-2243 (2016).

5. Bielawski, C. W. \& Grubbs, R. H. Living ring-opening metathesis polymerization. Prog. Polym, Sci. 32, 1-29 (2007).

6. Agard, N. J., Prescher, J. A. \& Bertozzi, C. R. A strain-promoted [3 + 2] azide-alkyne cycloaddition for covalent modification of biomolecules in living systems. J. Am. Chem. Soc. 126, 15046-15047 (2004).

7. Tokunaga, K., Sato, M., Kuwata, K., Miura, C., Fuchida, H., Matsunaga, N., Koyanagi, S., Ohdo, S., Shindo, N. \& Ojida, A. Bicyclobutane carboxylic amide as a cysteinedirected strained electrophile for selective targeting of proteins. J. Am. Chem. Soc. 142 , 18522-18531 (2020).

8. Turkowska, J., Durka, J. \& Gryko, D. Strain release - an old tool for new transformations. Chem. Commun. 56, 5718-5734 (2020).

9. Morten, C. J., Byers, J. A., Dyke, A. R. V., Vilotijevic, I. \& Jamison, T. F. The development of endo-selective epoxide-opening cascades in water. Chem. Soc. Rev. 38, 3175-3192 (2009).

10. Stirling, C. J. M. Some quantitative effects of strain on reactivity. Pure Appl. Chem. 56, 1781-1796 (1984).

11. Rablen, P. R. A Procedure for Computing Hydrocarbon Strain Energies Using Computational Group Equivalents, with Application to 66 Molecules. Chemistry 2, 347-360 (2020).

12. Earl, H. A., Marshall, D. R. \& Stirling, C. J. M. Eliminative ring fission of cyclobutanes: evaluation of acceleration by strain and the comparison with cyclopropanes. J. Chem. Soc., Chem. Commun. 779 (1983).

13. Sella, A., Basch, H. \& Hoz, S. Reactivity of strained compounds: Is ground state destabilization the major cause for rate enhancement? J. Am. Chem. Soc. 118, 416-420 (1996).

14. Sawicka, D., Wilsey, S. \& Houk, K. N. The $16 \mathrm{kcal} / \mathrm{mol}$ anomaly: Alteration of $[2+2+$ 2] cycloaddition rates by through-bond interactions. J. Am. Chem. Soc. 121, 864-865 (1999).

15. Verhoeven, J. W. Sigma-assistance; The modulation of intramolecular reactivity by through-bond interaction. Recl. Trav. Chim. Pays-Bas 99, 369-379 (1980).

16. Coulson, C. A. \& Moffitt, W. E. I. The properties of certain strained hydrocarbons. Lond. Edinb. Dubl. Phil. Mag. 40, 1-35 (1949).

17. Dewar, M. J. S. \& McKee, M. L. Aspects of Cyclic Conjugation. Pure Appl. Chem. 52, 1431-1441 (1980).

18. Weinhold, F. \& Landis, C. R. Valency and Bonding: A Natural Bond Orbital Donor-Acceptor Perspective. (Cambridge University Press, 2005).

19. Sterling, A. J., Dürr, A., Smith, R., Anderson, E. A. \& Duarte, F. Rationalizing the diverse reactivity of [1.1.1]propellane through $\sigma-\pi$-delocalization. Chem. Sci. 11, 4895-4903 (2020).

20. Burkhard, J. A., Wuitschik, G., Rogers-Evans, M., Müller, K. \& Carreira, E. M. Oxetanes as versatile elements in drug discovery and synthesis. Angew. Chem. Int. Ed. 49, 90529067 (2010).

21. Bull, J. A., Croft, R. A., Davis, O. A., Doran, R. \& Morgan, K. F. Oxetanes: Recent Advances in Synthesis, Reactivity, and Medicinal Chemistry. Chem. Rev. 116, 1215012233 (2016). 
22. Newcomb, E. T., Knutson, P. C., Pedersen, B. A. \& Ferreira, E. M. Total Synthesis of Gelsenicine via a Catalyzed Cycloisomerization Strategy. J. Am. Chem. Soc. 138, 108111 (2016).

23. Limanto, J. \& Snapper, M. L. Sequential Intramolecular Cyclobutadiene Cycloaddition, Ring-Opening Metathesis, and Cope Rearrangement: Total Syntheses of (+)- and (-)Asteriscanolide. J. Am. Chem. Soc. 122, 8071-8072 (2000).

24. Stepan, A. F., Subramanyam, C., Efremov, I. V., Dutra, J. K., O'Sullivan, T. J., DiRico, K. J., McDonald, W. S., Won, A., Dorff, P. H., Nolan, C. E., Becker, S. L., Pustilnik, L. R., Riddell, D. R., Kauffman, G. W., Kormos, B. L., Zhang, L., Lu, Y., Capetta, S. H., Green, M. E., Karki, K., Sibley, E., Atchison, K. P., Hallgren, A. J., Oborski, C. E., Robshaw, A. E., Sneed, B. \& O'Donnell, C. J. Application of the bicyclo[1.1.1]pentane motif as a nonclassical phenyl ring bioisostere in the design of a potent and orally active $\gamma$ secretase inhibitor. J. Med. Chem. 55, 3414-3424 (2012).

25. Pu, Q., Zhang, H., Guo, L., Cheng, M., Doty, A. C., Ferguson, H., Fradera, X., Lesburg, C. A., McGowan, M. A., Miller, J. R., Geda, P., Song, X., Otte, K., Sciammetta, N., Solban, N., Yu, W., Sloman, D. L., Zhou, H., Lammens, A., Neumann, L., Bennett, D. J., Pasternak, A. \& Han, Y. Discovery of Potent and Orally Available Bicyclo[1.1.1]pentane-Derived Indoleamine-2,3-dioxygenase 1 (IDO1) Inhibitors. ACS Med. Chem. Lett.11, 1548-1554 (2020).

26. Bell, R. P. The Theory of Reactions Involving Proton Transfers. Proc. R. Soc. Lond. 154, 414-429 (1936).

27. Evans, M. G. \& Polanyi, M. Inertia and Driving Force Of Chemical Reactions. J. Chem. Soc. Faraday Trans. 34, 11-24 (1938).

28. Marcus, R. A. On the Theory of Oxidation-Reduction Reactions Involving Electron Transfer. I. J. Chem. Phys. 24, 966-978 (1956).

29. Marcus, R. A. Theoretical relations among rate constants, barriers, and Broensted slopes of chemical reactions. J. Phys. Chem. 72, 891-899 (1968).

30. Enthalpies were chosen for a direct comparison with strain energies, which are commonly reported instead of Gibbs free energies. Trends in enthalpy and Gibbs free energy were found to be in excellent agreement for all reactions studied here.

31. Wolk, J. L., Hoz, T., Basch, H. \& Hoz, S. Quantification of the various contributors to rate enhancement in nucleophilic strain releasing reactions. J. Org. Chem. 66, 915-918 (2001).

32. Wolk, J. L., Sprecher, M., Basch, H. \& Hoz, S. Relative reactivity of three and four membered rings - The absence of charge effect. Org. Biomol. Chem. 2, 1065-1069 (2004).

33. Becke, A. D. \& Edgecombe, K. E. A simple measure of electron localization in atomic and molecular systems. J. Chem. Phys. 92, 5397-5403 (1990).

34. Gianatassio, R., Lopchuk, J. M., Wang, J., Pan, C.-M., Malins, L. R., Prieto, L., Brandt, T. A., Collins, M. R., Gallego, G. M., Sach, N. W., Spangler, J. E., Zhu, H., Zhu, J. \& Baran, P. S. Strain-release amination. Science 351, 241-246 (2016).

35. Sinninghe Damsté, J. S., Strous, M., Rijpstra, W. I. C., Hopmans, E. C., Geenevasen, J. A. J., van Duin, A. C. T., van Niftrik, L. A. \& Jetten, M. S. M. Linearly concatenated cyclobutane lipids form a dense bacterial membrane. Nature 419, 708-712 (2002).

36. Lopchuk, J. M., Fjelbye, K., Kawamata, Y., Malins, L. R., Pan, C.-M., Gianatassio, R., Wang, J., Prieot, L., Bradow, J., Brandt, T. A., Collins, M. R., Elleraas, J., Ewanicki, J., Farrell, W., Fadeyi, O. O., Gallego, G. M., Mousseau, J. J., Oliver, R., Sach, N. W., Smith, J. K., Spangler, J. E., Zhu, H., Zhu, J. \& Baran, P. S. Strain-Release Heteroatom Functionalization: Development, Scope, and Stereospecificity. J. Am. Chem. Soc.139, 3209-3226 (2017).

37. Wiberg, K. B., Waddell, S. T. \& Laidig, K. [1.1.1]Propellane: Reaction with free radicals. Tetrahedron Lett. 27, 1553-1556 (1986).

38. Wiberg, K. B. \& Waddell, S. T. Reactions of [1.1.1]Propellane. J. Am. Chem. Soc. 112, 2194-2216 (1990). 
39. Quantitative agreement was observed between the trend in activation enthalpies and free energies.

40. Liu, F., Paton, R. S., Kim, S., Liang, Y. \& Houk, K. N. Diels-Alder reactivities of strained and unstrained cycloalkenes with normal and inverse-electron-demand dienes: Activation barriers and distortion/interaction analysis. J. Am. Chem. Soc. 135, 15642-15649 (2013).

41. Singh, K., Fennell, C. J., Coutsias, E. A., Latifi, R., Hartson, S., Weaver, J. D. Light Harvesting for Rapid and Selective Reactions: Click Chemistry with Strain-Loadable Alkenes. Chem 4, 124-137 (2018).

42. Dommerholt, J., Rutjes, F. P. J. T. \& van Delft, F. L. Strain-Promoted 1,3-Dipolar Cycloaddition of Cycloalkynes and Organic Azides. Top. Curr. Chem. 374, 16 (2016).

43. Li, K., Fong, D., Meichsner, E. \& Adronov, A. A Survey of Strain-Promoted Azide-Alkyne Cycloaddition in Polymer Chemistry. Chem. Eur. J. 27, 5057-5073 (2021).

44. Blanksby, S. J. \& Ellison, G. B. Bond dissociation energies of organic molecules. Acc. Chem. Res. 36, 255-263 (2003).

45. Young, T. A., Silcock, J. J., Sterling, A. J. \& Duarte, F. autodE: Automated Calculation of Reaction Energy Profiles-Application to Organic and Organometallic Reactions. Angew. Chem. Int. Ed. 60, 4266-4274 (2021).

46. Riniker, S. \& Landrum, G. A. Better Informed Distance Geometry: Using What We Know to Improve Conformation Generation. J. Chem. Inf. Model. 55, 2562-2574 (2015).

47. Bannwarth, C., Ehlert, S. \& Grimme, S. GFN2-xTB - An Accurate and Broadly Parametrized Self-Consistent Tight-Binding Quantum Chemical Method with Multipole Electrostatics and Density-Dependent Dispersion Contributions. J. Chem. Theory Comput. 15, 1652-1671 (2019).

48. Weigend, F. \& Ahlrichs, R. Balanced Basis Sets of Split Valence, Triple Zeta Valence and Quadruple Zeta Valence Quality for H to Rn: Design and Assessment of Accuracy. Phys. Chem. Chem. Phys. 7, 3297-3305 (2005).

49. Grimme, S., Antony, J., Ehrlich, S. \& Krieg, H. A consistent and accurate ab initio parametrization of density functional dispersion correction (DFT-D) for the 94 elements HPu. J. Chem. Phys. 132, 154104 (2010).

50. Neese, F. Software update: the ORCA program system, version 4.0. Wiley Interdiscip. Rev. Comput. Mol. Sci. 8, 4-9 (2018).

51. Klopman, G. Solvations: a semi-empirical procedure for including solvation in quantum mechanical calculations of large molecules. Chem. Phys. Lett. 1, 200-202 (1967).

52. Barone, V. \& Cossi, M. Quantum Calculation of Molecular Energies and Energy Gradients in Solution by a Conductor Solvent Model. J. Chem. Phys. A 102, 1995-2001 (1998).

53. Marenich, A. v., Cramer, C. J. \& Truhlar, D. G. Universal solvation modle based on solute electron density and a contiuum model of the solvent defind by the bulk dielectric constant and atomic surface tensions. J. Phys. Chem. B. 113, 6378-6396 (2009).

54. Riplinger, C., Sandhoefer, B., Hansen, A. \& Neese, F. Natural triple excitations in local coupled cluster calculations with pair natural orbitals. J. Chem. Phys. 139, 134101 (2013).

55. Grimme, S. Semiempirical hybrid density functional with perturbative second-order correlation. J. Chem. Phys. 124, 034108 (2006).

56. Neese, F., Wennmohs, F., Hansen, A. \& Becker, U. Efficient, approximate and parallel Hartree-Fock and hybrid DFT calculations. A "chain-of-spheres" algorithm for the Hartree-Fock exchange. Chem. Phys. 356, 98-109 (2009).

57. Stoychev, G. L., Auer, A. A. \& Neese, F. Automatic Generation of Auxiliary Basis Sets. J. Chem. Theory Comput. 13, 554-562 (2017).

58. Lu, T. \& Chen, F. Multiwfn: A multifunctional wavefunction analyzer. J. Comput. Chem. 33, 580-592 (2012).

59. Pedregosa, F., Varoquaux, G., Gramfort, A., Michel, V., Thirion, B., Grisel, O., Blondel, M., Prettenhofer, P., Weiss, R., Dubourg, V., Vanderplas, J., Passos, A., Cournapeau, 
D., Brucher, M., Perrot, M. \& Duchesnay, É. Scikit-learn: Machine Learning in Python. J. Mach. Learn Res. 12, 2825-2830 (2011).

60. Hunter, J. D. Matplotlib: A 2D Graphics Environment. Comput. Sci. Eng. 9, 90-95 (2007). 